\title{
On real analytic functions of unbounded type
}

\author{
J.M. Ansemil · J. López-Salazar · S. Ponte
}

\begin{abstract}
In this paper we prove several results on the existence of analytic functions on an infinite dimensional real Banach space which are bounded on some given collection of open sets and unbounded on others. In addition, we also obtain results on the density of some subsets of the space of all analytic functions for natural locally convex topologies on this space.
\end{abstract}

\section{Introduction and definitions}

The space of holomorphic functions on a complex Banach space has attracted the attention of many researchers, who have produced a lot of interesting results; see, for instance, the list of references in [7]. However, that is not the case for real analytic functions. The reason is, among other things, that in the real case there is no Cauchy integral formula and the Taylor series expansion does not necessarily converge, even pointwise, on the biggest possible ball. Nevertheless, as we are going to see here, most of the results for complex analytic functions obtained in [1-4] and [8] can be also proved in the real case. To do this, we need that certain uniform lim- 
its on compact subsets of real analytic functions are analytic. While in the complex case the uniform limit on compact subsets of a sequence of holomorphic functions is also holomorphic, this is not true in the real case. As is well-known, any real periodic Lipschitz function on $\mathbb{R}$ (even not differentiable) is the uniform limit on $\mathbb{R}$ of its Fourier series, whose terms are analytic on $\mathbb{R}$. In addition, some other new results are obtained.

Definition 1 Let $X$ be a Banach space over $\mathbb{K}=\mathbb{R}$ or $\mathbb{C}$. Let $n \in \mathbb{N}$. A continuous mapping $P: X \rightarrow \mathbb{K}$ is an $n$-homogeneous polynomial if there is a symmetric $n$-linear continuous mapping $A: X^{n} \rightarrow \mathbb{K}$ such that $P(x)=A(x, \stackrel{n}{.}, x)$ for all $x \in X$. If $n=0$, the 0 -homogeneous polynomials are the constants. As usual, $\mathcal{P}\left({ }^{n} X\right)$ denotes the space of all $n$-homogeneous polynomials on $X$, while $\mathcal{P}(X)$ denotes the space of all polynomials on $X$, that is, finite sums of homogeneous polynomials.

Definition 2 Let $U$ be an open subset of $X$. A function $f: U \rightarrow \mathbb{K}$ is said to be analytic on $U$ if for every $x_{0} \in U$ there is a sequence $\left(P_{n}\right)_{n=0}^{\infty}$, where $P_{n} \in \mathcal{P}\left({ }^{n} X\right)$ for all $n$, such that

$$
f(x)=\sum_{n=0}^{\infty} P_{n}\left(x-x_{0}\right)
$$

uniformly on a neighborhood of $x_{0}$ contained in $U . \mathcal{A}(U)$ will denote the algebra of all analytic functions on $U$ and $\tau_{0}$ will be the compact open topology on $\mathcal{A}(U)$.

For the basic properties of real analytic functions see [5] or [12].

Each $P_{n}$ is the $n$-homogeneous polynomial associated to the $n^{\text {th }}$-derivative of $f$ at $x_{0}$. If $\mathbb{K}=\mathbb{C}$, the analytic functions are also called holomorphic and they are precisely the Fréchet differentiable functions on $U$ (see [10, Theorem 14.7]); in this case we write $\mathcal{H}(U)$ instead of $\mathcal{A}(U)$.

Definition 3 Let $X$ be a real Banach space with topological dual $X^{*}$. Let $\widetilde{X}$ denote the Cartesian product $X \times X$ with the operations

$$
(x, y)+\left(x^{\prime}, y^{\prime}\right)=\left(x+x^{\prime}, y+y^{\prime}\right), \quad(\alpha+i \beta)(x, y)=(\alpha x-\beta y, \alpha y+\beta x)
$$

for $(x, y),\left(x^{\prime}, y^{\prime}\right) \in X \times X, \alpha+i \beta \in \mathbb{C}$. The space $\widetilde{X}$ is a complex Banach space endowed with the Taylor's norm:

$$
\|(x, y)\|=\sup \left\{\sqrt{\varphi(x)^{2}+\varphi(y)^{2}}: \varphi \in X^{*},\|\varphi\|=1\right\} .
$$

Every real polynomial $P \in \mathcal{P}\left({ }^{n} X\right)$ has a unique complex extension $\widetilde{P} \in \mathcal{P}\left({ }^{n} \widetilde{X}\right)$, given by 


$$
\begin{aligned}
\widetilde{P}(x, y)= & \sum_{k=0}^{[n / 2]}(-1)^{k}\left(\begin{array}{c}
n \\
2 k
\end{array}\right) A\left(x^{n-2 k}, y^{2 k}\right) \\
& +i \sum_{k=0}^{[(n-1) / 2]}(-1)^{k}\left(\begin{array}{c}
n \\
2 k+1
\end{array}\right) A\left(x^{n-(2 k+1)}, y^{2 k+1}\right),
\end{aligned}
$$

where $A$ is the symmetric $n$-linear mapping associated to $P$ (see [9] or [11]). In particular, every $\varphi \in X^{*}$ can be extended to $\widetilde{X}$ by the formula

$$
\widetilde{\varphi}(x, y)=\varphi(x)+i \varphi(y) .
$$

It is easy to see that $\|\widetilde{\varphi}\|=\|\varphi\|$ (see [9, Proposition 3.1] or [11, Proposition 4]).

However, the situation concerning the extension of analytic functions is very different. It happens that not every analytic function on a real space $X$ can be extended to its complexification. A simple example is the function $f: \mathbb{R} \rightarrow \mathbb{R}, f(x)=\frac{1}{x^{2}+1}$, which is analytic on $\mathbb{R}$ but cannot be extended to an holomorphic function on $\mathbb{C}$.

\section{The results}

In this paper $X$ will always be a real Banach space of infinite dimension. If $S$ is a subset of $X$ and $f$ is a function on $S$, let $\|f\|_{S}=\sup \{|f(x)|: x \in S\}$. If $x \in X$ and $r>0$, let

$$
B(x, r)=\{y \in X:\|y-x\|<r\} \quad \text { and } \quad \widetilde{B}(x, r)=\{z \in \widetilde{X}:\|z-(x, 0)\|<r\} .
$$

Our aim here is to obtain some results on unbounded analytic functions on some open subsets of infinite dimensional real Banach spaces. The proofs, which need some particular techniques, will follow some ideas used in the proofs of similar results recently obtained in the complex case, most of them by the authors. See [1-3] and [8].

We start with a proposition which gives a sufficient condition for a real function to be analytic.

Proposition 1 Let $U$ be an open subset of $X$ and let $f$ be a real function on $U$. Assume that there is an open subset $\widetilde{U}$ of $\widetilde{X}$ such that $U \times\{0\} \subset \widetilde{U}$ and that there is a holomorphic extension $\widetilde{f}: \widetilde{U} \rightarrow \mathbb{C}$, that is, $\widetilde{f}(x, 0)=f(x)$ for all $x \in U$. Then $f$ is analytic on $U$.

Proof Let $x_{0} \in U$. There are $r>0$ and a sequence $\left(Q_{n}\right)_{n=0}^{\infty}$, where each $Q_{n}: \widetilde{X} \rightarrow \mathbb{C}$ is an $n$-homogeneous polynomial, such that

$$
\widetilde{f}(x, y)=\sum_{n=0}^{\infty} Q_{n}\left((x, y)-\left(x_{0}, 0\right)\right)
$$

uniformly on some $\widetilde{B}\left(x_{0}, r\right) \subset \widetilde{U}$. 
For $n \in \mathbb{N}$ and $x \in X$, let $P_{n}(x)=\operatorname{Re}\left(Q_{n}(x, 0)\right)$. Then $P_{n} \in \mathcal{P}\left({ }^{n} X\right)$. For every $x \in B\left(x_{0}, r\right)$ we have

$$
\begin{aligned}
\tilde{f}(x, 0) & =\sum_{n=0}^{\infty} Q_{n}\left((x, 0)-\left(x_{0}, 0\right)\right) \\
& =\sum_{n=0}^{\infty} \operatorname{Re}\left[Q_{n}\left((x, 0)-\left(x_{0}, 0\right)\right)\right]+i \sum_{n=0}^{\infty} \operatorname{Im}\left[Q_{n}\left((x, 0)-\left(x_{0}, 0\right)\right)\right] .
\end{aligned}
$$

Since $\widetilde{f}(x, 0)=f(x) \in \mathbb{R}$, necessarily

$$
f(x)=\sum_{n=0}^{\infty} \operatorname{Re}\left[Q_{n}\left((x, 0)-\left(x_{0}, 0\right)\right)\right]=\sum_{n=0}^{\infty} P_{n}\left(x-x_{0}\right)
$$

and this series converges uniformly on $B\left(x_{0}, r\right)$.

Proposition 2 Let $U$ be an open subset of $X$. The set of analytic functions on $U$ which can be extended to some open subset of $\widetilde{X}$ is $\tau_{0}$ dense in $\mathcal{A}(U)$.

Proof Every polynomial on $X$ can be extended to $\widetilde{X}$ and by the Stone-Weierstrass theorem, $\mathcal{P}(X)$ is $\tau_{0}$ dense in the space of all continuous functions on $U$, therefore in $\mathcal{A}(U)$.

In the proof of Theorems 1 and 4 we will use the next result on biorthogonal systems in Banach spaces, due to Dilworth, Girardi and Johnson:

Proposition 3 (See [6]) Let $x_{0} \in X$ and $\varphi_{0} \in X^{*}$. Then there are sequences $\left(x_{n}\right)_{n=1}^{\infty} \subset X$ and $\left(\varphi_{n}\right)_{n=1}^{\infty} \subset X^{*}$ with the following properties:

(a) $\lim _{n \rightarrow \infty} \varphi_{n}(x)=0$ for all $x \in X$,

(b) $\left\|\varphi_{n}\right\|=1$ for all $n \geq 1$,

(c) $\sup _{n \in \mathbb{N}}\left\|x_{n}\right\|<\infty$,

(d) $\varphi_{n}\left(x_{n}\right)=1$ for all $n \geq 1$,

(e) If $n, j \in \mathbb{N} \cup\{0\}$ and $n \neq j$, then $\varphi_{n}\left(x_{j}\right)=0$.

Theorem 1 If $B(0, R)$ and $B\left(x_{0}, r\right)$ are disjoint balls in $X$ and $\varepsilon>0$, then there is an analytic function $f: X \rightarrow \mathbb{R}$ with holomorphic extension $\widetilde{f}: \widetilde{X} \rightarrow \mathbb{C}$ such that

$$
\|\widetilde{f}\|_{\widetilde{B}(0, R)}<\varepsilon \quad \text { and } \quad\|f\|_{B\left(x_{0}, r\right)}=\infty .
$$

Proof By the Hahn-Banach theorem, there is $\varphi_{0} \in X^{*}$ such that $\left\|\varphi_{0}\right\|=1$ and $\varphi_{0}\left(x_{0}\right)=\left\|x_{0}\right\|$. Let $\left(x_{n}\right)_{n=1}^{\infty} \subset X$ and $\left(\varphi_{n}\right)_{n=1}^{\infty} \subset X^{*}$ be biorthogonal sequences with the properties given in Proposition 3. Let $C>\sup \left\|x_{n}\right\|$. For each $n \in \mathbb{N} \cup\{0\}$, let $\widetilde{\varphi}_{n} \in \widetilde{X}^{*}$ the complex extension of $\varphi_{n}$.

As $x_{0} \notin \bar{B}(0, R)$, then $\frac{R}{\left\|x_{0}\right\|}<1$, so there is $\alpha \in \mathbb{N}$ such that

$$
\left(\frac{R}{\left\|x_{0}\right\|}\right)^{\alpha}<\frac{r}{C R}
$$


Let $a>1$ such that

$$
\frac{C \cdot R^{\alpha+1}}{\left\|x_{0}\right\|^{\alpha} \cdot r}<\frac{1}{a}<1
$$

that is,

$$
a \frac{C}{\left\|x_{0}\right\|^{\alpha} \cdot r} \cdot R^{\alpha+1}<1
$$

As $\lim _{n \rightarrow \infty} \widetilde{\varphi}_{n}(z)=0$ for all $z \in \widetilde{X}$, the series

$$
\sum_{n=1}^{\infty}\left(a \frac{C}{\left\|x_{0}\right\|^{\alpha} \cdot r} \cdot \widetilde{\varphi}_{0}^{\alpha} \cdot \widetilde{\varphi}_{n}\right)^{n}
$$

converges uniformly on the compact subsets of $\widetilde{X}$. Therefore, it defines a holomorphic function $\widetilde{f}$ on $\widetilde{X}$. Consequently, by Proposition 1 ,

$$
f=\sum_{n=1}^{\infty}\left(a \frac{C}{\left\|x_{0}\right\|^{\alpha} \cdot r} \cdot \varphi_{0}^{\alpha} \cdot \varphi_{n}\right)^{n}
$$

is real analytic on $X$. On the one hand,

$$
\|\widetilde{f}\|_{\widetilde{B}(0, R)} \leq \sum_{n=1}^{\infty}\left(a \frac{C}{\left\|x_{0}\right\|^{\alpha} \cdot r} \cdot R^{\alpha+1}\right)^{n}<\infty .
$$

On the other hand,

$$
\begin{aligned}
\|f\|_{B\left(x_{0}, r\right)} & \geq \sup _{n \geq 1}\left|f\left(x_{0}+\frac{r}{C} x_{n}\right)\right| \\
& =\sup _{n \geq 1}\left(a \frac{C}{\left\|x_{0}\right\|^{\alpha} \cdot r} \cdot\left(\varphi_{0}\left(x_{0}\right)\right)^{\alpha} \cdot \frac{r}{C} \varphi_{n}\left(x_{n}\right)\right)^{n} \\
& =\sup _{n \geq 1} a^{n}=\infty .
\end{aligned}
$$

To finish, it suffices to take the functions

$$
\frac{\varepsilon}{\|\widetilde{f}\|_{\widetilde{B}(0, R)}+1} \widetilde{f} \text { and } \frac{\varepsilon}{\|\widetilde{f}\|_{\widetilde{B}(0, R)}+1} f .
$$

Definition 4 Let $U$ be an open subset of $X$. Let $I=\left(V_{n}\right)_{n=1}^{\infty}$ be an increasing open cover of $U$ and let

$$
\mathcal{A}_{I}(U)=\left\{f \in \mathcal{A}(U):\|f\|_{V_{n}}<\infty \text { for every } n\right\},
$$

endowed with the locally convex topology defined by the seminorms

$$
f \in \mathcal{A}_{I}(U) \mapsto\|f\|_{V_{n}}, \quad n \in \mathbb{N} .
$$


Note that $\mathcal{A}(U)$ is the union of the spaces $\mathcal{A}_{I}(U)$. Indeed, if $f \in \mathcal{A}(U)$, let $J=$ $\left(V_{n}\right)_{n=1}^{\infty}$, where

$$
V_{n}=\{x \in U:|f(x)|<n\}
$$

then $f \in \mathcal{A}_{J}(U)$.

The $\tau_{\delta}$ topology on $\mathcal{A}(U)$ is defined in the following way: $\left(\mathcal{A}(U), \tau_{\delta}\right)$ is the inductive limit of the spaces $\mathcal{A}_{I}(U)$, where $I$ ranges over the collection of all increasing countable open covers of $U$.

The $\tau_{\delta}$ topology was introduced by Nachbin [13] for the spaces of continuous functions. It is the bornological topology associated to $\tau_{0}$ on $\mathcal{A}(U)$.

The following theorem shows, in particular, that it is impossible to write $\mathcal{A}(U)$ as a countable union of spaces $\mathcal{A}_{I}(U)$ and also gives a result on density.

Theorem 2 Let $U$ be an open subset of $X$. For each $k \in \mathbb{N}$, let $I_{k}=\left(V_{k, j}\right)_{j=1}^{\infty}$ denote an increasing countable open cover of $U$. Then $\mathcal{A}(U) \neq \bigcup_{k=1}^{\infty} \mathcal{A}_{I_{k}}(U)$. Moreover,

$$
\mathcal{A}(U) \backslash \bigcup_{k=1}^{\infty} \mathcal{A}_{I_{k}}(U)
$$

is dense in $(\mathcal{A}(U), \tau)$ for every locally convex topology $\tau$ on $\mathcal{A}(U)$.

Proof Using a translation, we can assume that $0 \in U$. By [8, Proposition 7], there are sequences $\left(x_{k}\right)_{k=1}^{\infty} \subset U$ and $\left(R_{k}\right)_{k=0}^{\infty} \subset \mathbb{R}^{+}$such that

1. $\left(R_{k}\right)_{k=0}^{\infty}$ is increasing,

2. $U \subset \bigcup_{k=0}^{\infty} B\left(0, R_{k}\right)$,

3. $x_{k} \in B\left(0, R_{k}\right)$ and $x_{k} \notin \bar{B}\left(0, R_{k-1}\right)$ for every $k \geq 1$.

The collection $I_{1}=\left(V_{1, j}\right)_{j=1}^{\infty}$ is an open cover of $U$, so there is $j_{1} \in \mathbb{N}$ such that $x_{1} \in V_{1, j_{1}}$. Then there exists $r_{1}>0$ such that

$$
B\left(x_{1}, r_{1}\right) \subset V_{1, j_{1}}, \quad B\left(x_{1}, r_{1}\right) \subset B\left(0, R_{1}\right) \quad \text { and } \quad B\left(x_{1}, r_{1}\right) \cap B\left(0, R_{0}\right)=\varnothing
$$

By Theorem 1, there exists a function $f_{1} \in \mathcal{A}(X)$ with extension $\tilde{f}_{1} \in \mathcal{H}(\widetilde{X})$ such that

$$
\left\|\widetilde{f}_{1}\right\|_{\widetilde{B}\left(0, R_{0}\right)}<\frac{1}{2} \quad \text { and } \quad\left\|f_{1}\right\|_{B\left(x_{1}, r_{1}\right)}=\infty .
$$

$I_{2}=\left(V_{2, j}\right)_{j=1}^{\infty}$ is also an open cover of $U$, so there is $j_{2} \in \mathbb{N}$ such that $x_{2} \in V_{2, j_{2}}$. There exists $r_{2}>0$ such that

$$
B\left(x_{2}, r_{2}\right) \subset V_{2, j_{2}}, \quad B\left(x_{2}, r_{2}\right) \subset B\left(0, R_{2}\right), \quad B\left(x_{2}, r_{2}\right) \cap B\left(0, R_{1}\right)=\varnothing
$$

and

$$
\left\|f_{1}\right\|_{B\left(x_{2}, r_{2}\right)}<\infty
$$


(note that $f_{1}$ is continuous and then bounded on some ball centered in $x_{2}$ ). Again, there exists a function $f_{2} \in \mathcal{A}(X)$ with extension $\widetilde{f}_{2} \in \mathcal{H}(\widetilde{X})$ such that

$$
\left\|\widetilde{f}_{2}\right\|_{\widetilde{B}\left(0, R_{1}\right)}<\frac{1}{2^{2}} \text { and } \quad\left\|f_{2}\right\|_{B\left(x_{2}, r_{2}\right)}=\infty .
$$

By iterating this process, we get sequences $\left(j_{k}\right)_{k=1}^{\infty} \subset \mathbb{N},\left(r_{k}\right)_{k=1}^{\infty} \subset \mathbb{R}^{+},\left(f_{k}\right)_{k=1}^{\infty} \subset$ $\mathcal{A}(X),\left(\widetilde{f}_{k}\right)_{k=1}^{\infty} \subset \mathcal{H}(\widetilde{X})$, with the following properties:

(a) $B\left(x_{k}, r_{k}\right) \subset V_{k, j_{k}} \cap B\left(0, R_{k}\right)$,

(b) $\left\|f_{j}\right\|_{B\left(x_{k}, r_{k}\right)}<\infty$ if $j<k$,

(c) $\left\|\widetilde{f}_{k}\right\|_{\widetilde{B}\left(0, R_{k-1}\right)}<\frac{1}{2^{k}}$,

(d) $\left\|f_{k}\right\|_{B\left(x_{k}, r_{k}\right)}=\infty$,

(e) $f_{k}(x)=\widetilde{f}_{k}(x, 0)$ for all $x \in X$.

If $K$ is a compact subset of $\widetilde{U}:=\bigcup_{k=1}^{\infty} \widetilde{B}\left(0, R_{k}\right)$, then there is $k \in \mathbb{N}$ such that $K \subset \widetilde{B}\left(0, R_{k}\right)$ and thus

$$
\sum_{j=k+1}^{\infty}\left\|\widetilde{f}_{j}\right\|_{K} \leq \sum_{j=k+1}^{\infty}\left\|\widetilde{f}_{j}\right\|_{\widetilde{B}\left(0, R_{k}\right)} \leq \sum_{j=k+1}^{\infty}\left\|\widetilde{f}_{j}\right\|_{\widetilde{B}\left(0, R_{j-1}\right)} \leq \sum_{j=k+1}^{\infty} \frac{1}{2^{j}}<\infty .
$$

Therefore, the series $\sum_{j=1}^{\infty} \widetilde{f_{j}}$ converges uniformly on the compact subsets of $\widetilde{U}$. Since each $\widetilde{f}_{j}$ is holomorphic on $\widetilde{X}$, the function $\widetilde{f}=\sum_{j=1}^{\infty} \widetilde{f}_{j}$ is holomorphic on $\widetilde{U}$ so, according to Proposition $1, f: U \rightarrow \mathbb{R}$ given by

$$
f(x)=\widetilde{f}(x, 0)=\sum_{j=1}^{\infty} \widetilde{f}_{j}(x, 0)=\sum_{j=1}^{\infty} f_{j}(x)
$$

is analytic on $U$ (note that $U \times\{0\} \subset \widetilde{U}$ ).

Now we are going to prove that $f \notin \bigcup_{k=1}^{\infty} \mathcal{A}_{I_{k}}(U)$. Let $k \in \mathbb{N}$.

$$
\begin{aligned}
\|f\|_{V_{k, j_{k}}} & \geq\|f\|_{B\left(x_{k}, r_{k}\right)} \\
& \geq\left\|f_{k}\right\|_{B\left(x_{k}, r_{k}\right)}-\sum_{j=1}^{k-1}\left\|f_{j}\right\|_{B\left(x_{k}, r_{k}\right)}-\sum_{j=k+1}^{\infty}\left\|f_{j}\right\|_{B\left(x_{k}, r_{k}\right)} \\
& \geq\left\|f_{k}\right\|_{B\left(x_{k}, r_{k}\right)}-\sum_{j=1}^{k-1}\left\|f_{j}\right\|_{B\left(x_{k}, r_{k}\right)}-\sum_{j=k+1}^{\infty}\left\|\widetilde{f_{j}}\right\|_{\widetilde{B}\left(0, R_{j-1}\right)} .
\end{aligned}
$$

By (c), (d) and (e), we obtain that $\|f\|_{V_{k, j_{k}}}=\infty$, so $f$ does not belong to any of the spaces $\mathcal{A}_{I_{k}}(U)$.

Now we will see how $\mathcal{A}(U) \backslash \bigcup_{k=1}^{\infty} \mathcal{A}_{I_{k}}(U)$ is dense in $(\mathcal{A}(U), \tau)$ for every locally convex topology $\tau$ on $\mathcal{A}(U)$. For each pair of natural numbers $k$ and $j$, let

$$
W_{k, j}=V_{1, j} \cap V_{2, j} \cap \cdots \cap V_{k, j}
$$


The collection $J_{k}=\left(W_{k, j}\right)_{j=1}^{\infty}$ is an increasing countable open cover of $U$. Indeed, if $x \in U$, then there are $j_{1}, \ldots, j_{k} \in \mathbb{N}$ such that $x \in V_{1, j_{1}} \cap \cdots \cap V_{k, j_{k}}$, so

$$
x \in V_{1, j_{1}} \cap \cdots \cap V_{k, j_{k}} \subset V_{1, \max \left\{j_{1}, \ldots, j_{k}\right\}} \cap \cdots \cap V_{k, \max }\left\{j_{1}, \ldots, j_{k}\right\}=W_{k, \max \left\{j_{1}, \ldots, j_{k}\right\}} .
$$

If $k \in \mathbb{N}$ and $f \in \mathcal{A}_{I_{k}}(U)$, then $\|f\|_{V_{k, j}}<\infty$ for all $j$. Therefore $\|f\|_{W_{k, j}}<\infty$ for all $j$, so $f \in \mathcal{H}_{J_{k}}(U)$. This shows that $\mathcal{A}_{I_{k}}(U) \subset \mathcal{A}_{J_{k}}(U)$ and, consequently,

$$
\mathcal{A}(U) \backslash \bigcup_{k=1}^{\infty} \mathcal{A}_{J_{k}}(U) \subset \mathcal{A}(U) \backslash \bigcup_{k=1}^{\infty} \mathcal{A}_{I_{k}}(U) .
$$

Given a locally convex topology $\tau$ on $\mathcal{A}(U)$, we will prove that $\mathcal{A}(U) \backslash \bigcup_{k=1}^{\infty} \mathcal{A}_{J_{k}}(U)$ is dense in $(\mathcal{A}(U), \tau)$, so $\mathcal{A}(U) \backslash \bigcup_{k=1}^{\infty} \mathcal{A}_{I_{k}}(U)$ will be also dense.

Let $g \in \mathcal{A}(U), p$ be a continuous seminorm on $(\mathcal{A}(U), \tau)$ and $\varepsilon>0$. We can assume that $g$ does not belong to $\mathcal{A}(U) \backslash \bigcup_{k=1}^{\infty} \mathcal{A}_{J_{k}}(U)$. This means that there is $k_{1} \in \mathbb{N}$ such that $g \in \mathcal{A}_{J_{k_{1}}}(U)$, that is, $\|g\|_{W_{k_{1}, j}}<\infty$ for all $j$. If $k \geq k_{1}$, then $W_{k, j} \subset$ $W_{k_{1}, j}$, so $\|g\|_{W_{k, j}}<\infty$ for every $k \geq k_{1}$ and every $j \in \mathbb{N}$.

As we have seen above, $\mathcal{A}(U) \neq \bigcup_{k=1}^{\infty} \mathcal{A}_{J_{k}}(U)$, so there are $f \in \mathcal{A}(U)$ and $\left(j_{k}\right)_{k=1}^{\infty} \subset \mathbb{N}$ such that $\|f\|_{W_{k, j_{k}}}=\infty$ for every $k$. If $k \geq k_{1}$,

$$
\left\|\frac{\varepsilon}{p(f)+1} f+g\right\|_{W_{k, j_{k}}} \geq \frac{\varepsilon}{p(f)+1}\|f\|_{W_{k, j_{k}}}-\|g\|_{W_{k, j_{k}}}=\infty .
$$

If $k<k_{1}$, then $W_{k, j_{k_{1}}} \supset W_{k_{1}, j_{k_{1}}}$ and

$$
\left\|\frac{\varepsilon}{p(f)+1} f+g\right\|_{W_{k, j_{k}}} \geq\left\|\frac{\varepsilon}{p(f)+1} f+g\right\|_{W_{k_{1}, j_{k_{1}}}}=\infty .
$$

Therefore,

$$
h=\frac{\varepsilon}{p(f)+1} f+g \in \mathcal{A}(U) \backslash \bigcup_{k=1}^{\infty} \mathcal{A}_{J_{k}}(U) .
$$

As $p(h-g)<\varepsilon$, we obtain that $\mathcal{A}(U) \backslash \bigcup_{k=1}^{\infty} \mathcal{A}_{J_{k}}(U)$ is dense in $(\mathcal{A}(U), \tau)$.

The next theorem shows that there are real analytic functions that are arbitrarily small on a given ball and unbounded on a certain collection of balls.

Theorem 3 Let $\left(B_{n}\right)_{n=0}^{\infty}$ be a sequence of open balls in $X$ such that $B_{j} \nsubseteq B_{0}$ for every $j \in \mathbb{N}$. For each $j$, let

$$
s_{j}=\sup \left\{\|x\|: x \in B_{j}\right\}
$$

and assume that $\lim _{j \rightarrow \infty} s_{j}=\infty$. Then, given $\varepsilon>0$, there is a function $f \in \mathcal{A}(X)$ such that

$$
\|f\|_{B_{0}}<\varepsilon \quad \text { and } \quad\|f\|_{B_{j}}=\infty \quad \text { for every } j \in \mathbb{N} \text {. }
$$


Proof We may assume that $B_{0}=B\left(0, R_{0}\right)$ for some $R_{0}>0$. Since $\lim _{j \rightarrow \infty} s_{j}=\infty$, we can rearrange the sequence $\left(B_{j}\right)_{j=1}^{\infty}$ so that

$$
s_{1} \leq s_{2} \leq s_{3} \leq \cdots \text {. }
$$

Moreover, as each $B_{j}$ is an open set, then $\|x\|<s_{j}$ for every $x \in B_{j}$.

Let $x_{1} \in B_{1} \backslash \overline{B_{0}}$. Then

$$
R_{0}<\left\|x_{1}\right\|<s_{1},
$$

so there is $m_{1} \in \mathbb{N}$ such that $\left\|x_{1}\right\|<s_{1}-\frac{1}{m_{1}}$. We define $R_{1}=s_{1}-\frac{1}{m_{1}}$, which satisfies $R_{1}>R_{0}$. Since $R_{1}<s_{1} \leq s_{2}$, there is $x_{2} \in B_{2}$ such that

$$
R_{1}<\left\|x_{2}\right\|<s_{2}
$$

Again, we take $m_{2} \in \mathbb{N}, m_{2}>m_{1}$, such that $\left\|x_{2}\right\|<s_{2}-\frac{1}{m_{2}}$ and we define $R_{2}=$ $s_{2}-\frac{1}{m_{2}}$.

In this way, we get two sequences $\left(x_{j}\right)_{j=1}^{\infty} \subset X$ and $\left(R_{j}\right)_{j=0}^{\infty} \subset \mathbb{R}^{+}$with the following properties:

1. $\left(R_{j}\right)_{j=0}^{\infty}$ is increasing and $\lim _{j \rightarrow \infty} R_{j}=\infty$ because $\lim _{j \rightarrow \infty} s_{j}=\infty$,

2. For all $j \geq 1, x_{j} \in B_{j} \cap B\left(0, R_{j}\right)$ and $x_{j} \notin \bar{B}\left(0, R_{j-1}\right)$.

As in Theorem 2, there are sequences $\left(r_{j}\right)_{j=1}^{\infty} \subset \mathbb{R}^{+},\left(f_{j}\right)_{j=1}^{\infty} \subset \mathcal{A}(X)$ and $\left(\widetilde{f}_{j}\right)_{j=1}^{\infty} \subset \mathcal{H}(\widetilde{X})$ such that

3. $B\left(x_{j}, r_{j}\right) \subset B_{j} \cap B\left(0, R_{j}\right)$,

4. $\left\|f_{n}\right\|_{B\left(x_{j}, r_{j}\right)}<\infty$ if $1 \leq n \leq j-1$,

5. $\left\|\widetilde{f}_{j}\right\|_{\widetilde{B}\left(0, R_{j-1}\right)}<\frac{1}{2^{j}}$,

6. $\left\|f_{j}\right\|_{B\left(x_{j}, r_{j}\right)}=\infty$,

7. $f_{j}(x)=\widetilde{f_{j}}(x, 0)$ for all $x \in X$.

Again as in Theorem 2, the function $f=\sum_{n=1}^{\infty} f_{n}$ is real analytic on $X$, it is bounded on $B\left(0, R_{0}\right)$ and $\|f\|_{B_{j}} \geq\|f\|_{B\left(x_{j}, r_{j}\right)}=\infty$ for all $j \geq 1$. To complete the proof, it suffices to consider the function $\frac{\varepsilon}{\|f\|_{B_{0}}+1} f$.

The proof of the following proposition is similar to the proof of [3, Corollary 2.6], after one considers the complexification of $X$.

Proposition 4 Let $m, n \in \mathbb{N}$, with $n>m$, and let us consider two finite sequences of balls, $\left\{B\left(x_{i}, R_{i}\right)\right\}_{i=1}^{m}$ and $\left\{B\left(x_{j}, R_{j}\right)\right\}_{j=m+1}^{n}$, such that

$$
\max \left\{R_{1}, \ldots, R_{m}\right\}<\min \left\{R_{m+1}, \ldots, R_{n}\right\} .
$$

Then, for every $\varepsilon>0$, there is $f \in \mathcal{A}(X)$ such that

$$
\|f\|_{B\left(x_{i}, R_{i}\right)}<\varepsilon \quad \text { for all } i \in\{1, \ldots, m\}
$$

and

$$
\|f\|_{B\left(x_{j}, R_{j}\right)}=\infty \quad \text { for all } j \in\{m+1, \ldots, n\} .
$$


Proposition 5 Let us assume that $X$ has a Schauder basis $\left(e_{n}\right)_{n=1}^{\infty}$ such that

$$
0<\inf \left\|e_{n}\right\| \leq \sup \left\|e_{n}\right\|<\infty .
$$

Let $\left(\varphi_{n}\right)_{n=1}^{\infty} \subset X^{*}$ the sequence of orthogonal functionals associated to the basis and $M=\sup \left\|\varphi_{n}\right\|$. Given $J \subset \mathbb{N}, R_{j}>0$ for all $j \in J$ and $\varepsilon>0$, there is $f \in \mathcal{A}(X)$ such that

1. $\|f\|_{B\left(0, \frac{1}{M}\right)}<\varepsilon$,

2. $\|f\|_{B\left(e_{i}, \frac{1}{M}\right)}<\varepsilon$ for all $i \in \mathbb{N} \backslash J$,

3. $\|f\|_{B\left(e_{j}, R_{j}\right)}=\infty$ for all $j \in J$.

Proof For each $j \in J$, let $t_{j} \in \mathbb{R}$ such that

$$
1<t_{j}<1+\frac{R_{j}}{\left\|e_{j}\right\|} .
$$

Since $t_{j}>1$, there is $\alpha_{j} \in \mathbb{N}$ such that $\frac{1}{3} t_{j}^{\alpha_{j}} r_{j}>1$. Then by Proposition 1 the function

$$
f=\frac{\varepsilon}{5} \cdot \sum_{j \in J} \sum_{n=j+1}^{\infty}\left(\frac{1}{3} \varphi_{j}^{\alpha_{j}} \varphi_{n}\right)^{n}
$$

is analytic on $X$ and it satisfies the properties of the proposition. The details of the proof are as in [3, Theorem 2.7].

The next proposition gives a result on density on $\left(\mathcal{A}(X), \tau_{0}\right)$.

Proposition 6 Let $I$ and $J$ be disjoint subsets of $\mathbb{N}$. Let $\left\{B_{n}: n \in I \cup J\right\}$ be a collection of balls on $X$ such that $B_{j} \varsubsetneqq B_{i}$ for every $i \in I$ and $j \in J$. If there is a function $f \in \mathcal{A}(X)$ such that $\|f\|_{B_{i}}<\infty$ for all $i \in I$ and $\|f\|_{B_{j}}=\infty$ for all $j \in J$, then the set

$$
G=\left\{g \in \mathcal{A}(X):\|g\|_{B_{i}}<\infty \text { for all } i \in I \text { and }\|g\|_{B_{j}}=\infty \text { for all } j \in J\right\}
$$

is dense in $\left(\mathcal{A}(X), \tau_{0}\right)$.

Proof Let $h \in \mathcal{A}(X), \varepsilon>0$ and a compact subset $K \subset X$. By the Stone-Weierstrass theorem there is $P \in \mathcal{P}(X)$ such that $\|P-h\|_{K}<\frac{\varepsilon}{2}$. Then $g=\frac{\varepsilon}{2\|f\|_{K}+1} f+P \in G$ and $\|g-h\|_{K}<\varepsilon$.

This result can be applied in the cases given by Theorems 1 and 3, and Propositions 4 and 5.

Proposition 7 Let $\mathcal{A}_{b}(X)$ the set of all analytic functions on $X$ which are bounded on each bounded subset of $X$. Then there are an infinitely generated algebra $A \subset \mathcal{A}(X)$ and $a \tau_{0}$ dense subspace $W \subset \mathcal{A}(X)$ such that

$$
A \backslash\{0\} \subset \mathcal{A}(X) \backslash \mathcal{A}_{b}(X) \text { and } W \backslash\{0\} \subset \mathcal{A}(X) \backslash \mathcal{A}_{b}(X) .
$$


Proof Using Proposition 1, the proof is similar to those in [8, Theorems 2 and 5]. In this case, the density result for the $\tau_{0}$ topology follows from the density of $\mathcal{P}(X)$ in $\left(\mathcal{A}(X), \tau_{0}\right)$ by the Stone-Weierstrass theorem.

Our last theorem is a real version of a result by Aron [4], which has been the main motivation for most of the results in $[1-3,8]$ and in this paper. The part related with the density is proved for any locally convex topology on $\mathcal{A}(X)$.

We recall that the radius of boundedness of an analytic function at a point $x$ is defined as

$$
r_{b} f(x)=\sup \left\{r>0:\|f\|_{B(x, r)}<\infty\right\} .
$$

Theorem 4 There is an analytic function $f: X \rightarrow \mathbb{R}$ such that

$$
\inf \left\{r_{b} f(x):\|x\|=1\right\}=0 .
$$

Moreover, the set

$$
F=\{f \in \mathcal{A}(X): f \text { satisfies property (1) }\}
$$

is dense in $(\mathcal{A}(X), \tau)$ for every locally convex topology $\tau$ on $\mathcal{A}(X)$.

Proof Given a locally convex topology $\tau$ on $\mathcal{A}(X)$, let $p$ be a continuous seminorm on $(\mathcal{A}(U), \tau), g \in \mathcal{A}(X)$ and $\varepsilon>0$. We have to prove that there exists $f \in F$ such that $p(f-g)<\varepsilon$. We may assume that $g \notin F$, so there is $r>0$ such that $\|g\|_{B(x, r)}<\infty$ for all $x \in X,\|x\|=1$.

Let $\left(x_{n}\right)_{n=1}^{\infty} \subset X$ and $\left(\varphi_{n}\right)_{n=1}^{\infty} \subset X^{*}$ be biorthogonal sequences with the properties given in Proposition 3. The sequences $\left(y_{n}=\frac{x_{n}}{\left\|x_{n}\right\|}\right)_{n=1}^{\infty}$ and $\left(\psi_{n}=\left\|x_{n}\right\| \varphi_{n}\right)_{n=1}^{\infty}$ give another biorthogonal system, $\left\|y_{n}\right\|=1$ for every $n$ and $\lim _{n \rightarrow \infty} \psi_{n}(x)=0$ for all $x \in X$. For each $n \in \mathbb{N}$, let $\alpha_{n} \in \mathbb{N}$ such that $2^{\alpha_{n}} \frac{1}{n} \geq 2$.

For each $n \in \mathbb{N}$, let $\widetilde{\psi}_{n}$ be the complex extension of $\psi_{n}$. As $\lim _{n \rightarrow \infty} \widetilde{\psi}_{n}(z)=0$ for all $z \in \widetilde{X}$, the series

$$
\sum_{n=1}^{\infty} \sum_{k=n+1}^{\infty}\left(\left(2 \widetilde{\psi}_{n}\right)^{\alpha_{n}} \widetilde{\psi}_{k}\right)^{k}
$$

converges uniformly on the compact subsets of $\widetilde{X}$ and so it defines a holomorphic function $\tilde{f}$ on $\widetilde{X}$. Then $f: X \rightarrow \mathbb{R}$ given by $f(x)=\widetilde{f}(x, 0)$ is analytic on $X$.

Let $n \in \mathbb{N}, n \geq \frac{1}{r}$. On the one hand,

$$
\begin{aligned}
\|f\|_{B\left(y_{n}, \frac{1}{n}\right)} & =\|f\|_{\bar{B}\left(y_{n}, \frac{1}{n}\right)} \geq \sup _{k \geq n+1}\left|f\left(y_{n}+\frac{1}{n} y_{k}\right)\right| \\
& =\sup _{k \geq n+1}\left(2^{\alpha_{n}} \frac{1}{n}\right)^{k} \geq \sup _{k \geq n+1} 2^{k}=\infty .
\end{aligned}
$$

On the other hand,

$$
\|g\|_{B\left(y_{n}, \frac{1}{n}\right)} \leq\|g\|_{B\left(y_{n}, r\right)}<\infty .
$$




\section{Therefore,}

$$
\left\|\frac{\varepsilon}{p(f)+1} f+g\right\|_{B\left(y_{n}, \frac{1}{n}\right)} \geq\left\|\frac{\varepsilon}{p(f)+1} f\right\|_{B\left(y_{n}, \frac{1}{n}\right)}-\|g\|_{B\left(y_{n}, \frac{1}{n}\right)}=\infty,
$$

so

$$
r_{b}\left(\frac{\varepsilon}{p(f)+1} f+g\right)\left(y_{n}\right) \leq \frac{1}{n}
$$

for all $n \geq \frac{1}{r}$. This implies that $\frac{\varepsilon}{p(f)+1} f+g \in F$. Finally, $p\left(\left(\frac{\varepsilon}{p(f)+1} f+g\right)-g\right)<\varepsilon$. This completes the proof.

Acknowledgements The second author has been supported by Universidad Complutense de Madrid, grant BE45/08.

\section{References}

1. Ansemil, J.M., Aron, R.M., Ponte, S.: Representation of spaces of entire functions on Banach spaces. Publ. Res. Inst. Math. Sci. 45, 383-391 (2009)

2. Ansemil, J.M., Aron, R.M., Ponte, S.: Behavior of entire functions on balls in a Banach space. Indag. Math. 20, 483-489 (2009)

3. Ansemil, J.M., López-Salazar, J., Ponte, S.: Entire functions uniformly bounded on balls of a Banach space. Stud. Math. 204, 187-194 (2011)

4. Aron, R.M.: Entire functions of unbounded type on a Banach space. Boll. Unione Mat. Ital. 9, 28-31 (1974)

5. Bochnak, J.: Analytic functions on Banach spaces. Stud. Math. XXXV, 271-292 (1970)

6. Dilworth, S.J., Girardi, M., Johnson, W.B.: Geometry of Banach spaces and biorthogonal systems. Stud. Math. 140, 243-271 (2000)

7. Dineen, S.: Complex Analysis on Infinite Dimensional Spaces. Springer Monographs in Mathematics. Springer, London (1999)

8. López-Salazar, J.: Vector spaces of entire functions of unbounded type. Proc. Am. Math. Soc. 139, 1347-1360 (2011)

9. Kirwan, P.: Complexification of multilinear mappings and polynomials. Math. Nachr. 231, 39-68 (2001)

10. Mujica, J.: Complex Analysis in Banach Spaces. Dover, New York (2010)

11. Muñoz, G., Sarantopoulos, Y., Tonge, A.: Complexifications of real Banach spaces, polynomials and multilinear maps. Stud. Math. 134, 1-33 (1999)

12. Nachbin, L.: Lectures on the Theory of Distributions. Textos de Matemática, vol. 15. Instituto de Física e Matemática, Universidade do Recife, Recife (1964)

13. Nachbin, L.: Sur les espaces vectoriels topologiques d'applications continues. C. R. Acad. Sci. Paris, Sér. A 271, 596-598 (1970) 